\title{
The effect of lumbosacral manipulation on growing pains
}

\author{
Dawid de Beer ${ }^{1}$, Charmaine Margaret Bester* \\ University of Johannesburg, South Africa
}

\section{A R T I C L E I N F O}

Article history:

Received 12 February 2015

Accepted 28 April 2015

Available online 19 August 2015

Keywords:

Manipulation

Growing pain

Chiropractic

\begin{abstract}
A B S T R A C T
Background: Growing pains are a frequent clinical presentation that continues to puzzle practitioners, with very little conclusive evidence in any medical field, including chiropractic.

Objective: The aim of this study was to determine whether lumbosacral manipulations have an effect on growing pain symptoms.

Methods: Thirty participants with growing pains between the ages of 4 and 12 years were recruited. The participants were placed into two groups of 15 participants each. Group 1 received lumbosacral manipulations to restricted joints as determined by motion palpation, while Group 2 never received any professional intervention. Often parent(s)/guardian(s) of children who suffer from growing pains will rub the child's legs and offer verbal reassurance in an attempt to console their children. Parent(s)/guardian(s) of both groups were encouraged to continue to do this throughout the duration of the trial. Instructions were given to the parents so that the same rubbing technique and rubbing cream (aqueous cream) were used. Subjective changes were tracked using a pain diary that the parent(s)/ guardian(s) were asked to complete, a six-week post-study follow-up question regarding children's growing pains and the Oucher self-report pain scale. Objective measures consisted of pressure algometer readings of the tibialis anterior muscle belly.

Results: The statistical data was analysed using the Friedman test, Mann-Whitney test and the Wilcoxon Signed-Rank test. The results demonstrated that both groups responded favourably to their specific treatment over time. However, the group that received lumbosacral manipulations proved to show a quicker response to treatment; and the poststudy follow-up of this same group showed markedly more positive feedback than the group that did not receive the treatment. These results highlighted the positive effects of chiropractic manipulation on growing pain symptoms.

Conclusion: The results from this study, specifically the feedback from parent(s)/guardians(s) and the pain diaries, indicated that spinal manipulation is beneficial in the treatment of growing pains. The results also showed that other methods of treating growing pains, such as simple leg rubs, may also bring relief.
\end{abstract}

Copyright () 2015, The Authors. Production and hosting by Elsevier B.V. on behalf of Johannesburg University. This is an open access article under the CC BY-NC-ND license

(http://creativecommons.org/licenses/by-nc-nd/4.0/).

\footnotetext{
* Corresponding author. Tel.: +27 (0) 115596936.

E-mail addresses: dawiddebeer@celeris.co.za (D. de Beer), charmaineb@uj.ac.za (C.M. Bester).

${ }^{1}$ Tel.: +27 (0) 828261436.
}

Peer review under responsibility of Johannesburg University. 


\section{Introduction}

\subsection{Background}

French physician, Marcel Duchamp, first described growing pains (GP) in 1823 (Evans, 2008). According to Evans, Scutter, Lang, and Dansie (2006), Peterson provided the best definition in 1986 (Peterson, 1986). He defined GP by inclusion and exclusion criteria. The inclusion criteria were: intermittent pains in both legs (non-articular in location) that are generally present late in the day or at night time, often waking the individual. The exclusions were: physical signs (swelling, redness, trauma, reduced joint range, limping) and objective findings (blood tests, imaging).

\subsection{Prevalence}

According to Uziel and Hashkes (2007), growing pains diagnosed by typical clinical symptoms are the most common form of episodic childhood musculoskeletal pain occurring between the ages of 3 and 12 years. However, according to Lowe and Hashkes (2008), GP tend to occur in children aged 4-14 years. The prevalence of GP has been reported in nine separate studies since 1928 (Evans \& Scutter, 2004b). Evans and Scutter (2004b) have estimated the global prevalence of GP, as defined by Peterson, in children $4-6$ years of age to be at $36.9 \%$.

\subsection{Aetiology}

Many authors agree that there is no conclusive aetiology for GP (Al-Khattat \& Campbell, 2000; Evans, 2008; Evans \& Scutter, 2004a, 2007; Evans et al., 2006; Lowe \& Hashkes, 2008; Uziel \& Hashkes, 2007). Furthermore the term "growing pains" is thought to be a contradiction as there is no evidence that the process of growth is painful, the peak incidence of pain does not conincide with peak growth periods and pain does not occur at sites where growth is thought to take place (Lowe \& Hashkes, 2008).

Despite the uncertainty of the aetiology, three main theories dominate the literature - the anatomical, fatigability and psychological models (Evans, 2008). According to the anatomical theory, the cause of the leg pain is due to a postural or orthopaedic defect that could induce bad posture or stance and that treatment of the defect can be clinically observed to give relief (Evans \& Scutter, 2007). The fatigability theory is periodically mentioned and is based on the belief that there is an accumulation of metabolic waste products within the leg muscles; this theory, however, remains untested. The theory was developed since parents often associate episodes of GP with periods of increased physical activity (Evans et al., 2006). According to the psychological theory, increased vulnerability to pain is suspected, as well as a familial predisposition. There is dissent regarding gender bias, where girls have historically been regarded as more susceptible to GP than boys (Evans, 2008).

Chiropractors typically consider the anatomical (biomechanical) and pain referral aetiology, whereby pain from distant origins such as the lower back refer into the legs, as points where they could have an influence. According to Alcantara and Davis (2011), a chiropractic approach lends itself to supporting an anatomical aetiology of growing pains, albeit from a chiropractic perspective. It is thought that the solution lies in an understanding and appreciation of the biomechanical relationship between the spine, the pelvis and the lower extremities as this biomechanical relationship is bidirectional in nature.

\subsection{Management}

Evans et al. (2006) conducted a prevalence study in South Australia and found that approximately one-third (35.9\%) of parents sought professional advice concerning their child's GP condition. Of those who did, the majority consulted a doctor (26.8\%). Other health professionals consulted included chiropractors $(4.9 \%)$, podiatrists $(3.8 \%)$, and medical specialists (3.1\%). Only $5 \%$ of cases of the children taken to consult a health professional were investigated or treated.

There is no typical treatment prescribed in any of the presenting studies. However, different treatment options were sought and tried. Non-pharmacological approaches included were comforting and local massage therapy (Uziel \& Hashkes, 2007), muscle stretching (Evans, 2008), warmth modalities (Lowe \& Hashkes, 2008) or simply no management with general improvement over time (Uziel, Chapnick, Jaber, Nemet, \& Hashkes, 2010). Pharmacological approaches typically include analgesics such as paracetamol, chronic medication and various types of over-the-counter medication (Evans, 2008; Evans et al., 2006; Lowe \& Hashkes, 2008; Uziel \& Hashkes, 2007).

Joint manipulation has pain inhibitory effects that could relieve GP regardless of the cause, although this effect would be considered more management than curative of the problem. Mechanisms such as gate control whereby the stimulation of large diameter nerve fibres from normal tactile stimulation inhibit the pain felt from the smaller diameter nerve fibres that conduct pain could play a role in pain relief (Mendell, 2014). This mechanism would, however, also be activated with other physical therapies such as massage (Kessler, Marchant, \& Johnson, 2006). Manipulation also activates the descending pain inhibitory system from the dorsal periaqueductal (dPAG) gray (Skyba, Radhakrishnan, Rohlwing, Wright, \& Sluka, 2003; Sluka, Skyba, Radhakrishnan, Leeper, \& Wright, 2006). Wright (1995) demonstrated the effect of manipulation on this system by noting the specific responses of dPAG activation, most markedly being rapid analgesia. An increase in substance $P$, which has a potent analgesic effect, has also been shown to occur with joint manipulation (Molina-Ortega et al., 2014).

Despite the possible effects joint manipulation could have on GP, there is limited evidence on the efficacy of chiropractic manipulation as a treatment intervention. A few case studies have been published (Alcantara \& Davis, 2011; Fysh, 1992) which have reported favourable responses.

\subsection{Aim of the study}

This study aimed to assess the effect of chiropractic manipulation of lumbosacral joints found to be restricted during 
motion palpation on GP, combined with standard leg rubs and reassurance from the parent(s)/guardians(s). This was done by comparing a group of children receiving chiropractic manipulation to a control group that received only standard leg rubs and reassurance from the parent(s)/guardian(s).

\subsection{Contribution to field}

This study demonstrates the possible biomechanical link to GP and thereby offers more support to one of the many possible aetiologies associated with growing pains. The role played by chiropractic in pain inhibition may also be supported. Importantly, this study renders a proven treatment protocol for GP in the chiropractic profession and offers an option to parents with children suffering from GP.

\section{Research method and design}

\subsection{Design}

This was a quantitative, quasi experimental study examining 30 children diagnosed with GP. Treatment took place at the children's schools over a three-week treatment protocol consisting of two treatments per week. The hypothesis was that chiropractic manipulation would have a beneficial effect on the children's GP by its possible effect on biomechanics and pain inhibition.

\subsection{Materials}

The only material used in this study was a portable chiropractic table that was used to perform the lumbosacral manipulation.

\subsection{Recruitment procedure}

Children from primary and pre-schools in the Alberton and Bedfordview areas were informed of this study via an A5 advertisement insert in the schools' newsletters sent to the parent(s)/guardian(s). The researcher's contact details were on the letters so that interested participants could contact the researcher directly. The newsletters were sent to approximately 1000 learners in two primary schools and three preschools from which the first 30 participants that volunteered and qualified were recruited for the study. Only 30 participants were used as this study served to only demonstrate whether there was a change to motivate for more extensive studies on the topic. Potential participants who heard of this study via word of mouth could also participate.

\subsection{Inclusion and exclusion criteria}

Due to the lack of conclusive evidence regarding the aetiology of GP, the definition of GP for this study was: intermittent, bilateral leg pain that is non-articular in location and presents in the late afternoon without any physical abnormalities such as signs of trauma, skin lesions, congenital abnormalities, atypical bony alignment, etc. The participants were selected based on the inclusion/exclusion criteria described by Evans
(2008), these being bilateral, intermittent pain, localised to the musculature of the anterior thigh, calf and posterior knee, presenting in the late afternoon or early evening with normal findings on physical examination. Participants were excluded if they presented with any other description of pain or physical findings.

\subsection{Data collection method}

The 30 participants were divided into two groups of 15 each according to their entrance into the trial. The first 15 participants who volunteered to participate were allocated to Group 1 , and the remaining 15 children were allocated to Group 2. Group 1 received spinal manipulative therapy to the lumbosacral spine as well as leg rubs and consoling from the parent(s)/guardian(s) as per usual when needed. Group 2 received only leg rubs and consoling from the parent(s)/guardian(s) as per usual when needed.

Treatments and measurements took place in a private venue on the school grounds. The researcher performed all manipulations using diversified technique to manipulate restrictions found in the lumbosacral spine. A basic leg rub technique was explained and demonstrated to the parent(s)/ guardian(s) so that the same technique was used for both groups at home. It consisted of gentle rubbing in a circular pattern over the thighs and lower legs. The parent(s)/guardian(s) were requested to only use aqueous cream should they feel they needed a cream base for the leg rubs.

Group 1 received six spinal manipulative therapy treatments over a period of three weeks. The only objective measurements performed were the algometer measurements which were taken by the researcher on the first, third and fifth visit prior to treatment, and on the seventh visit. The algometry measurements were performed on the tibialis anterior muscle belly as children with GP have demonstrated a decreased pain threshold in this area (Lowe \& Hashkes, 2008). Subjective measurements consisted of the Oucher self-report pain scale (OSRPS), the pain diary and the 6-week post-trial follow-up question. The participants were required to complete the OSRPS on the first, third and fifth visit prior to treatment, and on the seventh visit. The OSRPS is a Likert item, whereby images of children with facial expressions of happy to sad are represented on a scale (Fig. 1). The children were requested to choose a face on the scale that best represented the GP they were experiencing. No treatment occurred at the seventh visit. The parent(s)/guardian(s) were required to hand in their pain diaries on the last visit.

Group 2 received no treatment from the researcher over the period of three weeks but had to come in for the same measurements as Group 1 four times at roughly five-day intervals.

The parent(s)/guardians(s) were requested to be present for at least the first and the last visits.

\subsection{Data analysis}

All statistical analysis was performed by STATKON (the statistical department at UJ). STATKON made use of the OSRPS results and the pressure pain threshold readings done with a pressure algometer. STATKON performed an exploratory data 


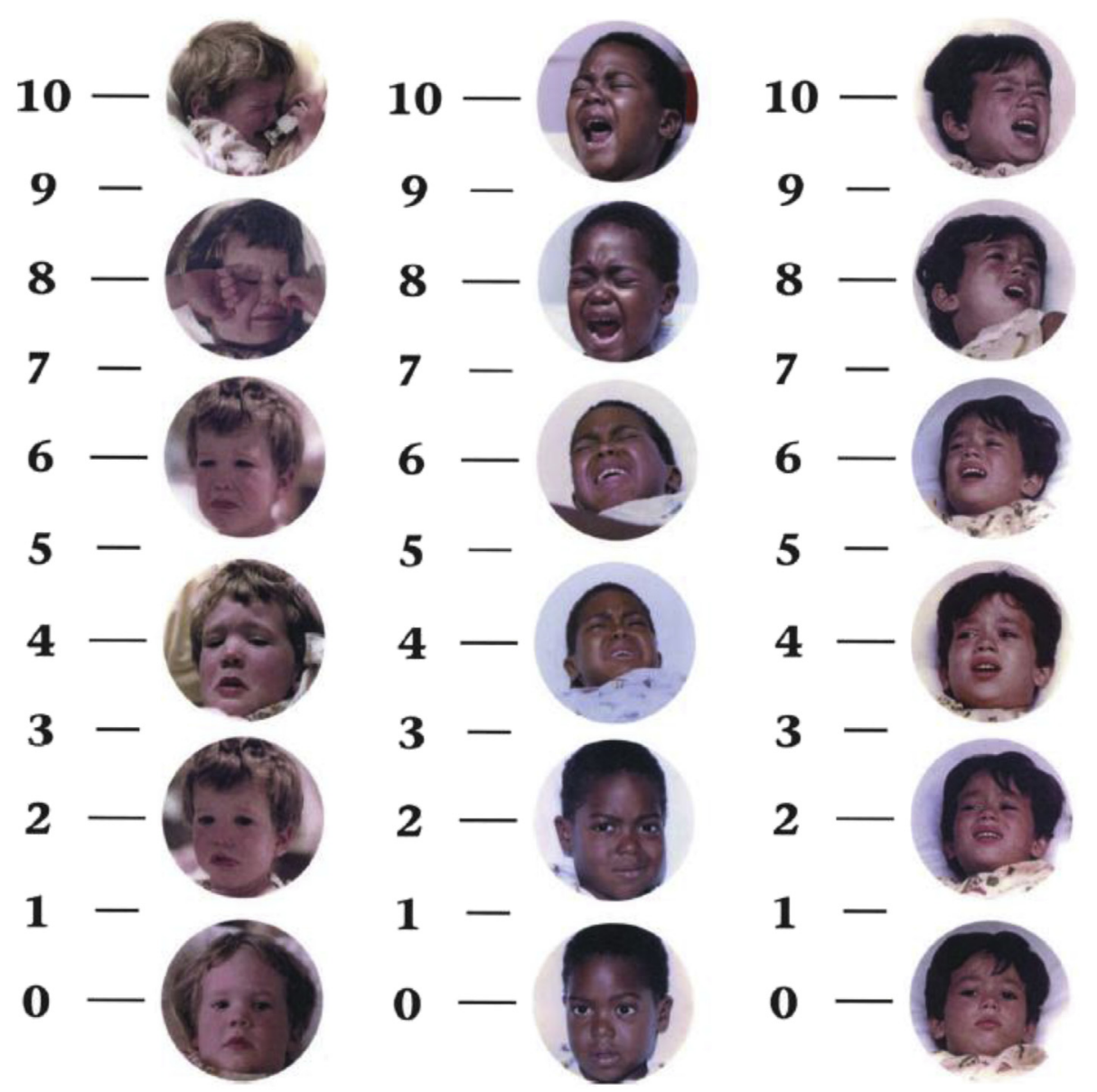

(Beyer, J., Villarruel, A., and Denyes, M., 2009).

Fig. 1 - Oucher self-report pain scale.

analysis (EDA) on the study results, looking at the normality and equal variances. EDA also assists in detection of mistakes, checking of assumptions, preliminary selection of appropriate models, determining relationships among the explanatory variables, and assessing the direction and rough size of relationships between explanatory and outcome variables. If the assumptions of normality and equal variances held true, parametric testing was used, and if not, non-parametric testing was used.

Parametric testing consisted of intergroup analysis making use of the independent samples t-test and intragroup analysis making use of repeated measures ANOVA. Independent samples t-test compared means for two groups, and repeated measures ANOVA tested the equality of means. Nonparametric testing consisted of intergroup analysis and made use of the Mann-Whitney $U$ test and intragroup analysis made use of the Friedman test. In the case of statistically significant findings, the Wilcoxon Signed-Rank test was performed.

\subsection{Context of study}

All children were recruited from the Alberton and Bedfordview areas which have similar socio-economic status and culture. The demographic distribution of the participants was thus a reflection of the demographics of the area.

\subsection{Ethical considerations}

The study was approved by the University of Johannesburg's Faculty of Health Sciences Higher Degrees Committee (HDC1801-2013) and Academic Ethics Committee (AEC18-01-2013). There were no major anticipated risks to the participants other than the possibility of slight post-manipulative pain and discomfort. As minors were involved in the study, consent as well as assent was obtained from the parent(s)/guardian(s) once the study was explained to the parent(s)/guardian(s) as well as the child. A child-friendly information form was given to the children making use of diagrams to assist in explaining the study. Privacy was ensured as all consultations took place in a private room provided by the school. Anonymity was maintained as no personal information was revealed on any of the data. Confidentiality was ensured by storing all data in a secured room with no unauthorised access. Participants were informed that participation was on a voluntary basis and that they could withdraw from the study at any stage.

The participants underwent an initial examination which determined the participants' suitability for the study. If any 
health concern was discovered during the examination, the participant was referred to the relevant healthcare professional. After examination, algometry measurements of the tibialis anterior were made and the OSRPS was completed. Participants were then treated according to their allocated groups. The participants in Group 2 were offered treatment post-study free of charge.

\section{Results}

\subsection{Pain diary}

Table 1 represents the values for the pain diaries completed and handed back at the end of the study. It can be seen that there was poor compliance on the initial pain diary and therefore no statistical analysis could be performed. The response to the post-study email was more favourable, although more so for Group 1.

\subsection{Post-study email}

A post-study follow-up was done six weeks after the study via email. The question posted to the parent(s)/guardian(s) of both groups was: "Have you noticed any changes or improvement in your child's growing pains or activities during the six weeks after the study?"

Group 1 reflected an overwhelmingly positive response with 12 parents/guardians giving positive responses, one giving what can be considered a neutral response, and two not responding at all. For Group 2 there was one negative response saying the growing pains got worse, four positive responses, and ten did not respond. See Table 2 for detailed feedback from the post-study follow-up question.

\section{OSRPS}

\subsection{Intragroup analysis}

The Friedman test was used to demonstrate any statistically significant improvement in the OSRPS readings between visit 1 and visit 7. Group 1 showed a p value of 0.002 and the $p$ value for Group 2 was 0.006 . Both groups showed $p$ values of $<0.05$, which indicates a statistically significant improvement in the perception of pain. The Wilcoxon Signed-Rank test was then performed to determine where this change occurred (see Table 3).

The Wilcoxon Signed-Rank test was used to demonstrate at which point any statistically significant improvement occurred ( $p<0.05$ ). Table 2 represents the statistical $p$ value results of the OSRPS scores using the Wilcoxon Signed-Rank

\begin{tabular}{lccc}
\hline Table 1 - Pain diaries. & & & \\
& Group 1 & Group 2 & Total \\
\hline Completed and returned & 9 & 1 & 10 \\
Not completed or returned & 6 & 14 & 20 \\
Total & 15 & 15 & 30 \\
\hline
\end{tabular}

Table 2 - Feedback (direct quotes) from post-study follow-up question: "Have you noticed any changes or improvement in your child's growing pains or activities during the six weeks after the study?".

Group 1

Positive responses

Neutral responses

Group 2

Positive responses

Neutral responses Negative responses
- We have experienced huge changes with ${ }^{* * *}{ }^{* * *}$ cried only once from bad growing pains after the study and not since then. It improved 100\%.

- At the moment the legs are not painful.

- It does feel as if ${ }^{* * *}$ complains less about painful legs.

- Thank you for your assistance. ${ }^{* * *}$ has not complained lately of any aches and pains. So, I can confidently say, yes, there has been a huge improvement.

- We found that while ${ }^{* * *}$ was treated during the study ${ }^{* * *}$ did complain a lot less of growing pains. We can recall one occasion. After the study ${ }^{* * *}$ did complain about two times of growing pains, definitely not as much as before the study.

- *** almost never complains about any pain in ${ }^{* * *}$ legs.

- With ${ }^{* * *}$ there was definitely an improvement. ${ }^{* * *}$ woke up a lot less from pain and had no pain in the afternoons. From 21/05/2013, ${ }^{* * *}$ has not complained of any pains. Something did definitely work!

- ${ }^{* * *}$ doesn't complain about pain in ${ }^{* * *}$ legs as constantly as before. ${ }^{* * *}$ runs and plays without any complaints.

- Had no pains after the study.

- Only had pain twice after the study.

- What I have picked up is that ${ }^{* * *}$ has not complained of any pain in the knees or shoulders, especially in the evenings.

- ${ }^{* * *}$ legs hasn't pained for a while now.

- *** started complaining again of pain in *** knees two weeks after the study.

- 2 no replies

- There was a decrease in growing pains.

- There was definitely an improvement with ***. The growing pains aren't so intense.

- After rubbing the legs three times a week, ${ }^{* * *}$ pain improved from bad to mild. ${ }^{* * *}$ does not wake up from pain any more but does still complain every now and then about pain.

- *** hasn't complained of any leg pain for a while now.

- 10 no replies

- *** growing pains are more intense, especially in the lower legs. As ${ }^{* * *}$ sleeps, ${ }^{* * *}$ cries and does not want stand on ${ }^{* * *}$ legs. test. Group 1 showed a significant change in the OSRPS rating right from the start with a $\mathrm{p}$ value of 0.007 from reading 1 to reading 2. Group 2 only started showing a significant change from reading 1 to reading 3 . 
Table 3 - Analysis of the OSRPS using the Wilcoxon Signed-Rank test.

\begin{tabular}{lcc} 
& Group 1 p value & Group 2 p value \\
\hline Reading 1-2 & 0.007 & 0.168 \\
Reading 1-3 & 0.044 & 0.031 \\
Reading 1-4 & 0.003 & 0.017 \\
Reading 2-3 & 0.671 & 0.301 \\
Reading 2-4 & 0.619 & 0.607 \\
Reading 3-4 & 0.530 & 0.937 \\
\hline
\end{tabular}

Table 4 - Analysis of the OSRPS using the

Mann-Whitney U test.

\begin{tabular}{lc} 
Reading & p Value \\
\hline 1 & 0.666 \\
2 & 0.121 \\
3 & 0.472 \\
4 & 0.319 \\
\hline
\end{tabular}

\subsection{Intergroup analysis}

Table 4 represents the statistical $p$ value results of the mean OSRPS scores using the Mann-Whitney test. The $p$ value for the first reading was 0.666 . As the $p$ value of the first reading was not $<0.05$, it shows no statistical significance and indicates that the two groups started off comparable. No statistical significance was noted between the two groups over the four readings.

\subsection{Pressure algometer}

Algometer readings of the anterior tibialis were taken as this has been shown to correlate with GP (Lowe \& Hashkes, 2008).

\subsection{Intragroup analysis}

The Friedman test was used to demonstrate any statistically significant improvement between visit 1 and visit 7 . Table 5 represents the statistical $\mathrm{p}$ value results of the pressure algometer readings using the Friedman test. Only Group 2 showed $p$ values $<0.05$, proving a statistically significant change. The Wilcoxon Signed-Rank test was then performed with the pressure algometer readings from Group 2 to determine where this change occurred. The Wilcoxon Signed-Rank test was used to demonstrate at which point any statistically significant improvement occurred.

\subsection{Intergroup analysis}

Table 6 represents the statistical p value results of the pressure algometer readings using the Mann-Whitney test. No statistical significance was noted between the two groups over the four readings in the right leg or the left leg.

Table 5 - Analysis of the pressure algometer readings using the Friedman test.

\begin{tabular}{cccccc} 
& \multicolumn{2}{c}{ Group 1 } & & \multicolumn{2}{c}{ Group 2 } \\
\cline { 2 - 3 } \cline { 5 - 6 } & Right leg & Left leg & & Right leg & Left leg \\
\hline p Value & 0.367 & 0.138 & & 0.001 & 0.000 \\
\hline
\end{tabular}

Table 6 - Analysis of the pressure algometer readings using the Mann-Whitney $U$ test.

\begin{tabular}{lcc} 
& \multicolumn{2}{c}{$\mathrm{p}$ Value } \\
\cline { 2 - 3 } & Right leg & Left leg \\
\hline 1 & 0.109 & 0.115 \\
2 & 0.533 & 0.170 \\
3 & 0.633 & 0.819 \\
4 & 0.350 & 0.262 \\
\hline
\end{tabular}

\section{Discussion}

\subsection{Outline of the results}

Although the aetiology of GP is unknown, the aetiological theories considered in this study, from a chiropractic perspective, were the anatomical (biomechanical) aetiology, pain referral aetiology, the activation of pain inhibitory systems and psychological impact or effects. Because the participants in Group 1 improved in subjective and objective measurements and the parent(s)/guardian(s) of the participants responded very positively to the post-study follow-up, one could consider the anatomical theory proposed by Evans and Scutter (2007) to be applicable in terms of which the cause of the leg pain may be due to a postural or an orthopaedic defect. It is possible that by treating the restrictions in the lumbar spine, nociceptor activation was decreased by relieving the mechanical stress induced by the joint restriction. These results can also be supported by the pain referral theory proposed by Cookson (2003). The activation of pain inhibitory systems might all also contribute to the aetiologies for GP and can also explain the favourable response of Group 1. Manipulation is thought to activate pain inhibitory systems via a few mechanisms such as gate control, activation of the dorsal periaqueductal gray descending inhibitory systems and release of substance $P$. This multi-system effect could explain the superior response to treatment in Group 1. However, it should be noted that all of these pain inhibitory mechanisms are thought to provide relatively short-term pain relief. Lastly, and possibly most importantly, the psychological impact also needs to be considered.

Although Group 1 showed the best and fastest improvement, both groups did improve despite Group 2 functioning as a control group. It was expected that Group 2 would show no improvement as no treatment beyond what the parents were already doing was performed. However, it was assumed that parents were already performing leg rubs and reassuring their children as this is the standard treatment for GP. It is possible that because GP have no known aetiology, parents may have considered rubbing their children's legs and providing reassurance as reinforcing pain behaviour. The data, however, indicates the opposite. By acknowledging the child's pain and providing a form of treatment, albeit very limited, the children in fact seemed to demonstrate a beneficial response. This could be explained via psychological and scientific reasoning whereby it could be said that the rubbing activated the pain inhibitory systems such as gate control. 


\subsection{Limitations}

Due to the small sample size and lack of response on the pain diary and post-study follow-up, statistical analysis could not be performed on this data. Assumptions and trends had to be used to interpret the data.

Although all measures used in this study have been tested scientifically for content and construct validity (Beyer, Denyes, \& Villarruel, 1992; Kinser, Sands, \& Stone, 2009), the researcher had some concerns. Some of the participants (particularly those under seven) did not seem to understand what growing pains were or what was expected from them regarding the OSRPS. Some participants tended to interpret any scratches or bruises on the legs as pain in the legs and would then complete the OSRPS accordingly. From age seven years and older, the researcher found good correlation between GP in the legs and the OSRPS readings. There was also some concern regarding the pressure algometer. Participants did not seem to understand the difference between pain threshold and pain tolerance. Many of the participants, again especially the younger participants, saw the pressure algometer as a game and the participants tried to see how much pain they could tolerate. The results from the above objective measures were nevertheless included as there was some valid data that could be interpreted; however, the above concerns need to be taken into account and the whole picture should be seen when interpreting the data.

The randomisation technique used to select participants did not allow for true random group allocation, and it is thus possible that the more eager parents/guardians or more severe cases volunteered first and were therefore placed in Group 1.

\subsection{Recommendations}

The results obtained in this study may be improved and validated by using a larger sample group whereby the population may be more accurately presented and allow for more contingency with regard to parent/guardian compliance where their feedback is needed. It would also be beneficial to conduct the study over a longer period, or to do further follow-up post-treatment as this would provide insight into whether the anatomical or pain inhibitory theories can be used to explain the positive effect. Chiropractic treatment beyond the lumbar spine would also provide further insights.

\section{Conclusion}

The results of the data collected from this study, the pressure algometer and OSRPS readings have shown that the spinal manipulation had some benefit. However, the pain diaries and feedback from the parents/guardians, which would probably be more reliable for GP, indicate that spinal manipulation provided significant improvement in terms of the children's perception of GP. The general trend noted among the children was a lower intensity and lower frequency of pain. There is a strong indication, despite the limitations of this study, that GP can be managed effectively with chiropractic treatment. However, the results also indicate that some relief may have occurred by simply reassuring the child and rubbing their legs. In conclusion, it appears that GP can be managed with relatively little intervention and need not be left for the child to "grow out of".

\section{R E F E R E N C E S}

Al-Khattat, A., \& Campbell, J. (2000). Recurrent limb pain in childhood ("growing pains"). The Foot, 10(3), 117-123.

Alcantara, J., \& Davis, J. (2011). The chiropractic care of children with "growing pains": a case series and systematic review of the literature. Complementary Therapies in Clinical Practice, 17, $28-32$.

Beyer, J. E., Denyes, M. J., \& Villarruel, A. M. (1992). The creation, validation, and continuing development of the Oucher: a measure of pain intensity in children. Journal of Pediatric Nursing, 7, 335-346.

Beyer, J., Villarruel, A., \& Denyes, M. (2009). User's manual and technical report (p. 2). The Oucher.

Cookson, L. (2003). Atypical knee pain: the biomechanical and neurological relationship between the pelvis, hip and knee - a case report. Clinical Chiropractic, 6, 63-66.

Evans, A. M. (2008). Growing pains: contemporary knowledge and recommended practice. Journal of Foot and Ankle Research, 1(4), 4.

Evans, A. M., \& Scutter, S. D. (2004a). Development of a questionnaire for parental rating of leg pain in young children: internal validity and reliability testing following triangulation. The Foot, 14(1), 42-48.

Evans, A. M., \& Scutter, S. D. (2004b). Prevalence of "growing pains" in young children. The Journal of Pediatrics, 145, 255-258.

Evans, A. M., \& Scutter, S. D. (2007). Are foot posture and functional health different in children with growing pains? Pediatrics International, 49(6), 991-996.

Evans, A. M., Scutter, S. D., Lang, M. G., \& Dansie, B. R. (2006). "Growing pains" in young children: a study of the profile, experiences and quality of life issues of four to six year old children with recurrent leg pain. The Foot, 16(3), 120-124.

Fysh, P. (1992). Growing pains. Dynamic Chiropractic, 10(19).

Kessler, J., Marchant, P., \& Johnson, M. I. (2006). A study to compare the effect of massage and static touch on experimentally induced pain in healthy volunteers. Physiotherapy, 92, 225-232.

Kinser, A. M., Sands, W. A., \& Stone, M. H. (2009). Reliability and validity of a pressure algometer. Journal of Strength and Conditioning Research, 23(1), 312-314.

Lowe, R. M., \& Hashkes, P. J. (2008). Growing pains: a noninflammatory pain syndrome of early childhood. Nature Clinical Practice Rheumatology, 4(10), 542-549.

Mendell, L. M. (2014). Constructing and deconstructing the gate theory of pain. Pain, 155, 210-216.

Molina-Ortega, F., Lomas-Vega, R., Hita-Contreras, F., Manzano, G. P., Achalandabaso, A., Ramos-Morcillo, A. J., et al (2014). Immediate effects of spinal manipulation on nitric oxide, substance $\mathrm{P}$ and pain perception. Manual Therapy, 19, 411-417.

Peterson, H. (1986). Growing pains. Pediatric Clinics of North America, 33, 1365-1372.

Skyba, D. A., Radhakrishnan, R., Rohlwing, J. J., Wright, A., \& Sluka, K. A. (2003). Joint manipulation reduces hyperalgesia by activation of monoamine receptors but not opiod or GABA receptors in the spinal cord. Pain, 106, 159-168.

Sluka, K. A., Skyba, D. A., Radhakrishnan, R., Leeper, B. J., \& Wright, A. (2006). Joint mobilization reduces hyperalgsia 
associated with chronic muscle and joint inflammation in rats. The Journal of Pain, 7(8), 602-607.

Uziel, Y., Chapnick, G., Jaber, L., Nemet, D., \& Hashkes, P. J. (2010). Five-year outcome of children with "growing pains": correlations with pain threshold. The Journal of Pediatrics, 156(5), 838-840.
Uziel, Y., \& Hashkes, P. J. (2007). Growing pains in children. Pediatric Rheumatology, 5(5), 5.

Wright, A. (1995). Hypoalgesia post-manipulative therapy: a review of a potential neurophysiological mechanism. Manual Therapy, 1, 11-16. 